\title{
Product associativity in scator algebras and the quantum wave function collapse
}

\author{
M. Fernández-Guasti ${ }^{*+}$ \\ ${ }^{a}$ Lab. de Óptica Cuántica, Departamento de Física, Universidad Autónoma Metropolitana - Iztapalapa, 09340 Ciudad de México, Ap. \\ postal. 55-534, Mexico \\ *Corresponding author E-mail: mfg@xanum.uam.mx \\ +Orcid: 0000-0002-1839-6002
}

\author{
Article Info \\ Keywords: Commutative algebras, $\mathrm{Hy}$ - \\ percomplex numbers, Non associative \\ algebras, Quantum measurement prob- \\ lem. \\ 2010 AMS: 30G35, $20 M 14$ \\ Received: 12 May 2018 \\ Accepted: 21 June 2018 \\ Available online: 26 June 2018
}

\begin{abstract}
The scator product in $1+n$ dimensions for $n>1$, is associative if all possible product pairs have a non vanishing additive scalar component. The product is in general, not associative in the additive representation whenever the additive scalar component of a product pair is zero. A particular case of this statement is non associativity due to zero products of non zero factors. These features of scator algebra could be used to model the quantum wave function evolution and collapse in a unified description.
\end{abstract}

\section{Introduction}

Scator algebras are $1+n$ dimensional algebras endowed with a main involution and an order parameter. The addition operation satisfies commutative group conditions in $\mathbb{R}^{1+n}$. The product operation, defined in a subset of $\mathbb{R}^{1+n}$, is always commutative and the conditions when it fulfills associativity are the matter of this communication. The scator product is not bilinear, thus, it cannot be represented as a matrix matrix product. For this same reason, it is in general not distributive over addition [6]. However, the scator set in $1+n$ dimensions, can be mapped into a higher dimensional space in order to recover distributivity. This procedure has been expounded for $1+2$ dimensional real scators by extending this set to 1+3 dimensional space [14]. The elements of scator algebra, decorated with an overhead oval, can be written as an ordered sequence of $1+n$ real numbers, $\stackrel{o}{\varphi}=\left(f_{0} ; f_{1}, \ldots, f_{j}, \ldots, f_{n}\right)$. The component with subindex zero, separated by a semicolon, has a distinct nature from the rest. In the additive representation, scator elements are described by a sum of components

$$
\stackrel{o}{\varphi}=\left(f_{0} ; f_{1}, \ldots, f_{j}, \ldots, f_{n}\right)=f_{0}+\sum_{j=1}^{n} f_{j} \mathbf{e}_{j},
$$

where $f_{0}, f_{j} \in \mathbb{R}$ for $j$ from 1 to $n$ in $\mathbb{N}$ and $\mathbf{e}_{j} \notin \mathbb{R}$. The component with subindex zero is labeled the additive scalar component whereas the subindices 1 to $n$ stand for the additive director components. This representation is similar to the rectangular form of complex numbers extended to higher dimensions. In some subsets of $\mathbb{R}^{1+n}$, there exists a multiplicative representation of scators that is akin to the polar form of complex or hyperbolic numbers extended to higher dimensional spaces [11]. This communication is restricted to associativity in the additive representation.

Product associativity in scator algebra has been loosely addressed in several previous communications [6, 9, 10, 11]. It has been correctly stated that the scator product in the additive representation is in general not associative if zero product pairs are involved. Zero products are products of non zero scator factors that yield a zero scator, i.e. a scator with all additive components equal to zero. However, it has been stated that the product is associative if zero products are avoided. This statement is not correct. When these assertions were made, it was not foreseen that there exist non associative products in the additive representation even when zero products are not involved. The decisive parameter in the additive representation is, as we shall presently see, whether the additive scalar component of any product pair is zero. In diverse graded algebras, notably algebra of physical space (APS) [3], space-time algebra (STA) [13] and commutative quaternions [4], the scalar component has been used to represent time [5]. We propose that the collapse of the wave function in quantum mechanics may be 
described via the non associativity of the scator product. The reduction occurs when the time variable, represented by the additive scalar component, is zero. If each director component constitutes a quantum state, when the scalar component of the product becomes null, all but one (single state) of the director components of the product become zero.

Sufficient conditions for the scator product associativity are addressed in section 2, while section 3, evaluates the necessary conditions for the lack of associativity. The possibility of scator algebra to describe the wavefunction evolution and collapse is outlined in section 4 . The appendix deals with associativity exceptions. The remaining paragraphs in this introduction establish the necessary algebra prerequisites.

\section{Product operation}

We denote the scator set by

$$
\mathbb{S}^{1+n}=\left\{\stackrel{o}{\varphi} \in \mathbb{R}^{1+n}: f_{0} \neq 0, \text { if there exists } f_{j} f_{l} \neq 0 \text { for } j \neq l \in n\right\},
$$

the subset of $\mathbb{R}^{1+n}$ where the additive scalar is different from zero if two or more director components are not zero. This set is the union of two disjoint subsets

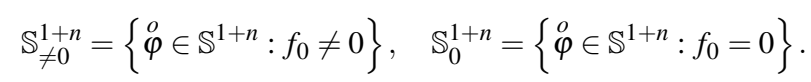

The elements of the set $\mathbb{S}_{0}^{1+n}$ have at most only one non vanishing director component, that is elements of the form $\left(0 ; 0, \ldots, f_{l}, \ldots, 0\right)=f_{l} \mathbf{e}_{l}$,

$$
\mathbb{S}_{l}^{1+n}=\left\{\stackrel{o}{\varphi} \in \mathbb{S}^{1+n}: f_{0}=0, f_{j}=0 \text { for all } j \neq l \text { from } 1 \text { to } n\right\}
$$

The set $\mathbb{S}_{0}^{1+n}$ can be written as the union of all subsets with at most one non vanishing director component $\mathbb{S}_{0}^{1+n}=\bigcup_{l=1}^{n} \mathbb{S}_{l}^{1+n}$. The sets $\mathbb{S}_{l}^{1+n}$ are not disjoint since the element with zero components everywhere is common to all of them, $\cap_{l=1}^{n} \mathbb{S}_{l}^{1+n}=(0 ; 0, \ldots, 0, \ldots, 0)=\stackrel{o}{0}$. In the additive representation, the scator product is defined in the $\mathbb{S}^{1+n}$ set:

The product of two scators $\stackrel{o}{\alpha}=a_{0}+\sum_{j=1}^{n} a_{j} \mathbf{e}_{j}$ and $\stackrel{o}{\beta}=b_{0}+\sum_{j=1}^{n} b_{j} \mathbf{e}_{j}$ in $\mathbb{S}_{\neq 0}^{1+n}$ is defined by

$$
\stackrel{o}{\gamma=\stackrel{o}{\alpha} \beta}=\underbrace{a_{0} b_{0} \prod_{k=1}^{n}\left(1 \mp \frac{a_{k} b_{k}}{a_{0} b_{0}}\right)}_{g_{0}}+\underbrace{a_{0} b_{0} \sum_{j=1}^{n} \prod_{k \neq j}^{n}\left(1 \mp \frac{a_{k} b_{k}}{a_{0} b_{0}}\right)\left(\frac{a_{j}}{a_{0}}+\frac{b_{j}}{b_{0}}\right)}_{g_{j}} \mathbf{e}_{j} ;
$$

If $\stackrel{o}{\alpha} \in \mathbb{S}_{l}^{1+n}$ and $\stackrel{o}{\beta} \in \mathbb{S}_{\neq 0}^{1+n}$ the product $\stackrel{o}{\alpha} \beta$ is

$$
\left(a_{l} \mathbf{e}_{l}\right) \stackrel{o}{\beta}=\mp a_{l} b_{l}+a_{l} b_{0} \mathbf{e}_{l} \mp \sum_{j \neq l}^{n}\left(a_{l} \frac{b_{l} b_{j}}{b_{0}}\right) \mathbf{e}_{j} ;
$$

If $\stackrel{o}{\alpha} \in \mathbb{S}_{l}^{1+n}$ and $\stackrel{o}{\beta} \in \mathbb{S}_{m}^{1+n}$,

$$
\left(a_{l} \mathbf{e}_{l}\right)\left(b_{m} \mathbf{e}_{m}\right)=\mp a_{l} b_{m} \delta_{l m},
$$

where $\delta_{l m}$ is a Kroneker delta.

The scator product defined in the $\mathbb{S}^{1+n}$ set is closed, commutative and there is a multiplicative identity [6]. Existence of an inverse depends on the signature of the product. Wherever applicable, here and in the rest of this manuscript, the upper $\mp$ (negative) sign corresponds to the imaginary scators product while the lower (positive) sign corresponds to the real or hyperbolic scators product. From (1.2c), $\check{\mathbf{e}}_{j} \check{\mathbf{e}}_{j}=-1$ for imaginary scators, we usually label the unit imaginary director components with a check above. When the product is defined with the (upper) negative sign, the $1+1$ dimensional scator sets with scalar component and only one director component, become isomorphic to complex algebra. In contrast, $\hat{\mathbf{e}}_{j} \hat{\mathbf{e}}_{j}=1$ for real scators, their director unit components usually labeled with a hat above. Real scators in $1+1$ dimensions are isomorphic to hyperbolic numbers. The check/hat decoration is omitted here in order to cope with both scator sets simultaneously.

\section{Associativity in the additive representation}

In the proofs that follow, products with three scator factors are evaluated. Products with a larger number of factors can be obtained by induction.

\subsection{Conditions for a product with non vanishing additive scalar component}

From the product definition (1.2a), the product of two scators with non zero additive scalar components $\left(a_{0} b_{0} \neq 0\right)$, has a non vanishing scalar component, if and only if

$$
\frac{a_{k} b_{k}}{a_{0} b_{0}} \neq \pm 1
$$

for all $k$ from 1 to $n$. From (1.2b), the product of non zero factors, one with zero scalar component $\stackrel{o}{\alpha}=a_{l} \mathbf{e}_{l}$, times $\stackrel{o}{\beta} \in \mathbb{S}_{\neq 0}^{1+n}$, has a non zero additive scalar component, if and only if $b_{l} \neq 0$. If both scator factors have null scalar, from (1.2c), their product has non zero scalar only if both scators have the same non zero director component, $l=m$. In the derivations that follow, it is assumed that none of the initial scator factors is zero. 


\subsection{Associative conditions}

Theorem 2.1. The scator product in $\mathbb{S}^{1+n}$ is associative in the additive representation if all possible product pairs have a non vanishing additive scalar component, $(\stackrel{o}{\alpha} \stackrel{o}{\beta}) \stackrel{o}{\varphi}=\stackrel{o}{\alpha}(\stackrel{o}{\beta} \stackrel{o}{\varphi})=\stackrel{o}{\beta}(\stackrel{o}{\alpha} \stackrel{o}{\varphi})$ if $\stackrel{o}{\alpha} \stackrel{o}{\beta}, \stackrel{o}{\beta} \stackrel{o}{\varphi}, \stackrel{o}{\alpha} \stackrel{o}{\varphi} \in \mathbb{S}_{\neq 0}^{1+n}$.

Proof. Case i) Scator factors with non zero additive scalar, $\stackrel{o}{\alpha}, \stackrel{o}{\beta}, \stackrel{o}{\varphi} \in \mathbb{S}_{\neq 0}^{1+n}$.

The product of scators $\stackrel{o}{\gamma}=\stackrel{o}{\alpha} \beta$ with $\stackrel{o}{\alpha, \beta}, \stackrel{o}{\beta} \in \mathbb{S}_{\neq 0}^{1+n}$ is given by (1.2a). Since all product pairs must have non zero scalar, $\stackrel{o}{\alpha \beta} \beta \in \mathbb{S}_{\neq 0}^{1+n}$; then $\frac{a_{k} b_{k}}{a_{0} b_{0}} \neq \pm 1$ for all $k$ from 1 to $n$. The subsequent product with $\stackrel{o}{\varphi} \in \mathbb{S}_{\neq 0}^{1+n}$ is again given by (1.2a),

$$
\begin{aligned}
(\stackrel{o}{\alpha} \beta) \stackrel{o}{\varphi} & =a_{0} b_{0} \prod_{k=1}^{n}\left(1 \mp \frac{a_{k} b_{k}}{a_{0} b_{0}}\right) f_{0} \prod_{k=1}^{n}\left(1 \mp \frac{\left(\frac{a_{k}}{a_{0}}+\frac{b_{k}}{b_{0}}\right)}{\left(1 \mp \frac{a_{k} b_{k}}{a_{0} b_{0}}\right)} \frac{f_{k}}{f_{0}}\right) \\
& +a_{0} b_{0} \prod_{k=1}^{n}\left(1 \mp \frac{a_{k} b_{k}}{a_{0} b_{0}}\right) f_{0} \sum_{j=1}^{n} \prod_{k \neq j}^{n}\left(1 \mp \frac{\left(\frac{a_{k}}{a_{0}}+\frac{b_{k}}{b_{0}}\right)}{\left(1 \mp \frac{a_{k} b_{k}}{a_{0} b_{0}}\right)} \frac{f_{k}}{f_{0}}\right)\left(\frac{\left(\frac{a_{j}}{a_{0}}+\frac{b_{j}}{b_{0}}\right)}{\left(1 \mp \frac{a_{j} b_{j}}{a_{0} b_{0}}\right)}+\frac{f_{j}}{f_{0}}\right) \mathbf{e}_{j} .
\end{aligned}
$$

This expression can be written in a symmetrical form in the coefficients of the three scators,

$$
\begin{aligned}
(\stackrel{o}{\alpha} \beta) \stackrel{o}{\varphi} & =a_{0} b_{0} f_{0} \prod_{k=1}^{n}\left(1 \mp \frac{a_{k} b_{k}}{a_{0} b_{0}} \mp \frac{a_{k} f_{k}}{a_{0} f_{0}} \mp \frac{b_{k} f_{k}}{b_{0} f_{0}}\right) \\
& +a_{0} b_{0} f_{0} \sum_{j=1}^{n} \prod_{k \neq j}^{n}\left(1 \mp \frac{a_{k} b_{k}}{a_{0} b_{0}} \mp \frac{a_{k} f_{k}}{a_{0} f_{0}} \mp \frac{b_{k} f_{k}}{b_{0} f_{0}}\right)\left(\frac{a_{j}}{a_{0}}+\frac{b_{j}}{b_{0}}+\frac{f_{j}}{f_{0}} \mp \frac{a_{j} b_{j} f_{j}}{a_{0} b_{0} f_{0}}\right) \mathbf{e}_{j} .
\end{aligned}
$$

The first factor in parenthesis can be grouped back as $\left(1 \mp \frac{b_{k} f_{k}}{b_{0} f_{0}} \mp\left(\frac{b_{k}}{b_{0}}+\frac{f_{k}}{f_{0}}\right) \frac{a_{k}}{a_{0}}\right)$, whereas the last factors are grouped as $\left(\frac{b_{j}}{b_{0}}+\frac{f_{j}}{f_{0}}+\left(1 \mp \frac{b_{j} f_{j}}{b_{0} f_{0}}\right) \frac{a_{j}}{a_{0}}\right)$. But $\stackrel{o}{\beta} \stackrel{o}{\varphi} \in \mathbb{S}_{\neq 0}^{1+n}$, thus, the terms $1 \mp \frac{b_{k} f_{k}}{b_{0} f_{0}} \neq 0$ for all $k$ from 1 to $n$, can be factored to obtain

$$
\begin{aligned}
(\stackrel{o}{\alpha} \stackrel{o}{\beta}) \stackrel{o}{\varphi} & =a_{0} b_{0} f_{0} \prod_{k=1}^{n}\left(1 \mp \frac{b_{k} f_{k}}{b_{0} f_{0}}\right)\left(1 \mp \frac{\left(\frac{b_{k}}{b_{0}}+\frac{f_{k}}{f_{0}}\right)}{\left(1 \mp \frac{b_{k} f_{k}}{b_{0} f_{0}}\right.} \frac{a_{k}}{a_{0}}\right) \\
& +b_{0} f_{0} \prod_{k=1}^{n}\left(1 \mp \frac{b_{k} f_{k}}{b_{0} f_{0}}\right) a_{0} \sum_{j=1}^{n} \prod_{k \neq j}^{n}\left(1 \mp \frac{\left(\frac{b_{k}}{b_{0}}+\frac{f_{k}}{f_{0}}\right)}{\left(1 \mp \frac{b_{k} f_{k}}{b_{0} f_{0}}\right)} \frac{a_{k}}{a_{0}}\right)\left(\frac{\frac{b_{j}}{b_{0}}+\frac{f_{j}}{f_{0}}}{\left(1 \mp \frac{b_{j} f_{j}}{b_{0} f_{0}}\right)}+\frac{a_{j}}{a_{0}}\right) \mathbf{e}_{j}=\stackrel{o}{\alpha}(\stackrel{o}{\beta} \stackrel{o}{\varphi}) .
\end{aligned}
$$

Since the product is always commutative, the factorization, provided that $\frac{a_{k} f_{k}}{a_{0} f_{0}} \neq \pm 1$, can also be performed to construct $\stackrel{o}{\beta}(\stackrel{o}{\alpha} \stackrel{o}{\varphi})$. Therefore, the product is associative for scator factors with non zero scalar components $a_{0} b_{0} f_{0} \neq 0$, if all three possible product pairs have non zero scalar component $\frac{a_{j} b_{j}}{a_{0} b_{0}} \neq \pm 1, \frac{b_{j} f_{j}}{b_{0} f_{0}} \neq \pm 1, \frac{a_{j} f_{j}}{a_{0} f_{0}} \neq \pm 1$, for all $j$ from 1 to $n$.

Case ii) Two scator factors have a non zero additive scalar, $\stackrel{o}{\alpha}, \stackrel{o}{\beta} \in \mathbb{S}_{\neq 0}^{1+n}$ and one factor has zero additive scalar component, $\stackrel{o}{\varphi} \in \mathbb{S}_{l}^{1+n}$. Product $(\stackrel{o}{\alpha} \stackrel{o}{\beta}) \stackrel{o}{\varphi}$. The product of scators $\stackrel{o}{\alpha, \beta}, \stackrel{o}{\beta}$ is given by $(1.2 \mathrm{a})$, where $\stackrel{o}{\alpha} \stackrel{o}{\beta} \in \mathbb{S}_{\neq 0}^{1+n}$ since their product must have non zero scalar. The product with a scator having zero scalar component $\stackrel{o}{\varphi}=f_{l} \mathbf{e}_{l}$, from $(1.2 \mathrm{~b})$ is then

$$
\begin{aligned}
(\stackrel{o}{\alpha} \beta) f_{l} \mathbf{e}_{l} & =\mp f_{l} a_{0} b_{0} \prod_{k \neq l}^{n}\left(1 \mp \frac{a_{k} b_{k}}{a_{0} b_{0}}\right)\left(\frac{a_{l}}{a_{0}}+\frac{b_{l}}{b_{0}}\right)+f_{l} a_{0} b_{0} \prod_{k=1}^{n}\left(1 \mp \frac{a_{k} b_{k}}{a_{0} b_{0}}\right) \mathbf{e}_{l} \\
& \mp \sum_{j \neq l}^{n}\left(f_{l} \frac{a_{0} b_{0} \prod_{k \neq l}^{n}\left(1 \mp \frac{a_{k} b_{k}}{a_{0} b_{0}}\right)\left(\frac{a_{l}}{a_{0}}+\frac{b_{l}}{b_{0}}\right)\left(\frac{a_{j}}{a_{0}}+\frac{b_{j}}{b_{0}}\right)}{\left(1 \mp \frac{a_{j} b_{j}}{a_{0} b_{0}}\right)}\right) \mathbf{e}_{j} .
\end{aligned}
$$

Product $\stackrel{o}{\alpha}(\stackrel{o}{\beta} \stackrel{o}{\varphi})$. The product $\stackrel{o}{\beta} \stackrel{o}{\varphi}$ is obtained from $(1.2 \mathrm{~b})$, where $f_{l} b_{l}$ should not be zero in order to have $\stackrel{o}{\beta} \stackrel{o}{\varphi} \in \mathbb{S}_{\neq 0}^{1+n}$. The product with $\stackrel{o}{\alpha}$ is given by (1.2a) since both factors have non zero scalar. The resulting expression can be simplified to

$$
\begin{aligned}
\stackrel{o}{\alpha}\left(\stackrel{o}{\beta} f_{l} \mathbf{e}_{l}\right) & =\mp f_{l} b_{l} a_{0} \prod_{k \neq l}^{n}\left(1 \mp \frac{a_{k} b_{k}}{a_{0} b_{0}}\right)\left(1+\frac{a_{l} b_{0}}{b_{l} a_{0}}\right) \mp f_{l} b_{l} a_{0}\left[\prod_{k \neq l}^{n}\left(1 \mp \frac{a_{k} b_{k}}{a_{0} b_{0}}\right)\left(\frac{a_{l}}{a_{0}} \mp \frac{b_{0}}{b_{l}}\right)\right] \mathbf{e}_{l} \\
& \mp f_{l} b_{l} a_{0} \sum_{j \neq l}^{n}\left[\prod_{k \neq j, l}^{n}\left(1 \mp \frac{a_{k} b_{k}}{a_{0} b_{0}}\right)\left(1+\frac{a_{l} b_{0}}{b_{l} a_{0}}\right)\left(\frac{a_{j}}{a_{0}}+\frac{b_{j}}{b_{0}}\right)\right] \mathbf{e}_{j} .
\end{aligned}
$$

Comparison component by component of (2.3) and (2.4) shows that both expressions are identical. Evaluation of $\stackrel{o}{\beta}(\stackrel{o}{\alpha} \stackrel{o}{\varphi})$ by a similar procedure shows that the three product associations give the same result.

Case iii) One scator factor has non zero additive scalar, $\stackrel{o}{\varphi} \in \mathbb{S}_{\neq 0}^{1+n}$, and two factors have zero scalar component, $\stackrel{o}{\alpha}, \stackrel{o}{\beta} \in \mathbb{S}_{0}^{1+n}$. 
The scalar component of the product $\stackrel{o}{\alpha} \stackrel{o}{\beta}=\left(a_{l} \mathbf{e}_{l}\right)\left(b_{k} \mathbf{e}_{k}\right)=\mp a_{l} b_{k} \delta_{l k}$ is not zero only if $k=l$, thus $\stackrel{o}{\beta}=b_{l} \mathbf{e}_{l}$.

$\operatorname{Product}(\stackrel{o}{\alpha} \stackrel{o}{\beta}) \stackrel{o}{\varphi}$. The product of $\stackrel{o}{\alpha} \stackrel{o}{\beta}=\mp a_{l} b_{l}$ times a scator $\stackrel{o}{\varphi} \in \mathbb{S}_{\neq 0}^{1+n}$ is $(\stackrel{o}{\alpha} \stackrel{o}{\beta}) \stackrel{o}{\varphi}=\mp a_{l} b_{l}\left(f_{0}+\sum_{j=1}^{n} f_{j} \mathbf{e}_{j}\right)$.

Product $\stackrel{o}{\alpha}(\stackrel{o}{\beta} \stackrel{o}{\varphi})$. The product $\stackrel{o}{\beta} \stackrel{o}{\varphi}$, is given by (1.2b), where the scalar component of this product is not zero if $f_{l} \neq 0$. Multiplication by $\stackrel{o}{\alpha}=a_{l} \mathbf{e}_{l}$, again from (1.2b), gives

$$
\stackrel{o}{\alpha}(\stackrel{o}{\beta} \stackrel{o}{\varphi})=a_{l} \mathbf{e}_{l}\left(b_{l} \mathbf{e}_{l}^{o} \stackrel{\varphi}{\varphi}\right)=a_{l} b_{l}\left(\mp f_{0}+\left(\mp f_{l}\right) \mathbf{e}_{l} \mp \sum_{j \neq l}^{n}\left(\frac{f_{0}}{\mp f_{l}}\right)\left(\mp \frac{f_{l} f_{j}}{f_{0}}\right) \mathbf{e}_{j}\right) .
$$

Thus, $(\stackrel{o}{\alpha} \stackrel{o}{\beta}) \stackrel{o}{\varphi}=\stackrel{o}{\alpha}(\stackrel{o}{\beta} \stackrel{o}{\varphi})$. Evaluation of $\stackrel{o}{\beta}(\stackrel{o}{\alpha} \stackrel{o}{\varphi})$ shows that all three associations are equal.

Case iv) All scator factors have zero scalar, $\stackrel{\circ}{\alpha}, \stackrel{o}{\beta}, \stackrel{o}{\varphi} \in \mathbb{S}_{0}^{1+n}$.

The scalar components of the products $\stackrel{o}{\alpha} \stackrel{o}{\beta}=\left(a_{l} \mathbf{e}_{l}\right)\left(b_{k} \mathbf{e}_{k}\right)=\mp a_{l} b_{k} \delta_{l k}, \stackrel{o}{\beta} \stackrel{o}{\varphi}=\left(b_{k} \mathbf{e}_{k}\right)\left(f_{j} \mathbf{e}_{j}\right)=\mp b_{k} f_{j} \delta_{k j}, \stackrel{o o}{\alpha} \stackrel{o}{\varphi}=\left(a_{l} \mathbf{e}_{l}\right)\left(f_{j} \mathbf{e}_{j}\right)=\mp a_{l} f_{j} \delta_{l j}$, are not zero only if $k=l=j$, thus $(\stackrel{o o}{\alpha} \stackrel{o}{\beta}) \stackrel{o}{\varphi}=\stackrel{o}{\alpha}(\stackrel{o}{\beta} \stackrel{o}{\varphi})=\stackrel{o}{\beta}(\stackrel{o}{\alpha} \stackrel{o}{\varphi})=\mp a_{j} b_{j} f_{j} \mathbf{e}_{j}$.

Notice that associativity holds even if the factors have zero additive scalar provided that all product pairs have non vanishing scalar. Theorem 2.1 establishes sufficient but not necessary conditions for the product associativity in the additive representation. Particular cases where the product is associative, although there are product pairs with zero scalar component, are examined in the appendix.

\section{Lack of associativity in the additive representation}

\subsection{Conditions for the product to yield a zero additive scalar component}

Factors with non vanishing scalar. Consider the product of two factors $\stackrel{o}{\alpha}, \stackrel{o}{\beta} \in \mathbb{S}_{\neq 0}^{1+n}$, from $(1.2 \mathrm{a})$,

$$
\begin{aligned}
\stackrel{o}{\alpha} \beta & =a_{0} b_{0} \prod_{k \neq l}^{n}\left(1 \mp \frac{a_{k} b_{k}}{a_{0} b_{0}}\right)\left(1 \mp \frac{a_{l} b_{l}}{a_{0} b_{0}}\right)+a_{0} b_{0} \prod_{k \neq l}^{n}\left(1 \mp \frac{a_{k} b_{k}}{a_{0} b_{0}}\right)\left(\frac{a_{l}}{a_{0}}+\frac{b_{l}}{b_{0}}\right) \mathbf{e}_{l} \\
& +a_{0} b_{0} \sum_{j \neq l}^{n}\left[\prod_{k \neq j, l}^{n}\left(1 \mp \frac{a_{k} b_{k}}{a_{0} b_{0}}\right)\left(1 \mp \frac{a_{l} b_{l}}{a_{0} b_{0}}\right)\left(\frac{a_{j}}{a_{0}}+\frac{b_{j}}{b_{0}}\right)\right] \mathbf{e}_{j}
\end{aligned}
$$

where the $l^{\text {th }}$ director component has been written out explicitly. Let this product have zero scalar component due to the $l^{\text {th }}$ factor,

$$
\left(1 \mp \frac{a_{l} b_{l}}{a_{0} b_{0}}\right)=0, \Longleftrightarrow \frac{b_{l}}{b_{0}}= \pm \frac{a_{0}}{a_{l}}
$$

A necessary condition for this expression to hold is that $a_{l}$ and $b_{l}$ should not be zero. Then, the product $\stackrel{o o}{\alpha} \beta \in \mathbb{S}_{l}^{1+n}$ has only one non vanishing director component given by

$$
\stackrel{\circ}{\alpha} \beta=a_{0} b_{0} \prod_{k \neq l}^{n}\left(1 \mp \frac{a_{k} b_{k}}{a_{0} b_{0}}\right)\left(\frac{a_{l}}{a_{0}} \pm \frac{a_{0}}{a_{l}}\right) \mathbf{e}_{l}
$$

One factor has vanishing scalar. If $\stackrel{o}{\alpha} \in \mathbb{S}_{l}^{1+n}$ and $\stackrel{o}{\beta} \in \mathbb{S}_{\neq 0}^{1+n}$, from (1.2b), this product has zero scalar if $a_{l} b_{l}=0$. Since the factors are not zero, $a_{l} \neq 0$, then the product has zero scalar component if $b_{l}=0$ and is given by $\stackrel{o}{\alpha} \stackrel{o}{\beta}=\left(a_{l} \mathbf{e}_{l}\right) \stackrel{o}{\beta}=a_{l} b_{0} \mathbf{e}_{l}$. Notice that this product is never zero for non zero scator factors, a result that is of paramount importance when evaluating differential quotients [8].

Both factors have vanishing scalar From (1.2c), $\left(a_{l} \mathbf{e}_{l}\right)\left(b_{j} \mathbf{e}_{j}\right)=\mp a_{l} b_{j} \delta_{l j}$. Only if $j \neq l$, the additive scalar is zero.

Zero products For scator factors with non vanishing scalar, if two or more factors are zero in expression (1.2a),

$$
\left(1 \mp \frac{a_{l} b_{l}}{a_{0} b_{0}}\right)=0,\left(1 \mp \frac{a_{m} b_{m}}{a_{0} b_{0}}\right)=0 \text { for } l \neq m,
$$

the additive scalar and all director components are zero, $\stackrel{o}{\alpha \beta}=\stackrel{o}{=}=0$. For scator factors with vanishing scalar, the scator product is zero only if $j \neq l, \stackrel{o}{\alpha} \stackrel{o}{\beta}=\left(a_{l} \mathbf{e}_{l}\right)\left(b_{j} \mathbf{e}_{j}\right)=0$. The zero product condition, where non associativity has been asserted before [6, 11, 12], will now be encompassed in the condition where a scator product pair with zero additive scalar component is involved. Besides the zero products, the product can also be zero if any of the factors is zero. This trivial case is dismissed since only non zero factors are considered as mentioned before. 


\subsection{Non associative conditions}

Theorem 3.1. The scator product in $\mathbb{S}^{1+n}$ is in general not associative for $n>1$ in the additive representation, if one or more of the possible product pairs has a vanishing additive scalar component, $(\stackrel{o}{\alpha} \stackrel{o}{\beta}) \stackrel{o}{\varphi} \neq \stackrel{o}{\alpha}(\stackrel{o}{\beta} \stackrel{o}{\varphi}) \neq \stackrel{o}{\beta}(\stackrel{o}{\alpha} \stackrel{o}{\varphi})$ if $\stackrel{o}{\alpha} \stackrel{o}{\beta}$ or $\stackrel{o}{\beta} \stackrel{o}{\varphi}$ or $\stackrel{o}{\alpha} \stackrel{o}{\varphi} \in \mathbb{S}_{0}^{1+n}$.

Proof. Case 1. Product of three factors with non zero scalar component, $\stackrel{o}{\alpha}, \stackrel{o}{\beta}, \stackrel{o}{\varphi} \in \mathbb{S}_{\neq 0}^{1+n}$.

Subcase 1.1 Only one product pair has a vanishing scalar component, $\stackrel{o}{\alpha} \stackrel{o}{\beta} \in \mathbb{S}_{0}^{1+n}, \stackrel{o}{\beta} \stackrel{o}{\varphi}, \stackrel{o o}{\alpha} \stackrel{o}{\varphi} \in \mathbb{S}_{\neq 0}^{1+n}$.

Product $(\stackrel{o}{\alpha} \stackrel{o}{\beta}) \stackrel{o}{\varphi}$. The $\stackrel{o}{\gamma}=\stackrel{o}{\alpha} \stackrel{o}{\beta} \in \mathbb{S}_{l}^{1+n}$ product is given by (3.2) and its product with a scator $\stackrel{o}{\varphi} \in \mathbb{S}_{\neq 0}^{1+n}$ is then given by $(1.2 \mathrm{~b})$,

$$
(\stackrel{o}{\alpha} \stackrel{o}{\beta}) \stackrel{o}{\varphi}=a_{0} b_{0} \prod_{k \neq l}^{n}\left(1 \mp \frac{a_{k} b_{k}}{a_{0} b_{0}}\right)\left(\frac{a_{l}}{a_{0}} \pm \frac{a_{0}}{a_{l}}\right)\left(\mp f_{l}+f_{0} \mathbf{e}_{l} \mp \sum_{j \neq l}^{n}\left(\frac{f_{l} f_{j}}{f_{0}}\right) \mathbf{e}_{j}\right) .
$$

This result is not symmetric in the three scators variables, thus already indicative of the lack of associativity, but let us not anticipate. Product $\stackrel{o}{\alpha}(\stackrel{o}{\beta} \stackrel{o}{\varphi})$. Evaluate $\stackrel{o}{\beta} \stackrel{o}{\varphi} \in \mathbb{S}_{\neq 0}^{1+n}$ from (1.2a). The product with $\stackrel{o}{\alpha}$ using (1.2a) again, is then

$$
\begin{aligned}
\stackrel{o}{\alpha}(\stackrel{o}{\beta} \stackrel{o}{\varphi}) & =a_{0} b_{0} f_{0} \prod_{k=1}^{n}\left(1 \mp \frac{b_{k} f_{k}}{b_{0} f_{0}}\right)\left(1 \mp \frac{\left(\frac{b_{k}}{b_{0}}+\frac{f_{k}}{f_{0}}\right)}{\left(1 \mp \frac{b_{k} f_{k}}{b_{0} f_{0}}\right)} \frac{a_{k}}{a_{0}}\right) \\
& +b_{0} f_{0} \prod_{k=1}^{n}\left(1 \mp \frac{b_{k} f_{k}}{b_{0} f_{0}}\right) a_{0} \sum_{j=1}^{n} \prod_{k \neq j}^{n}\left(1 \mp \frac{\left(\frac{b_{k}}{b_{0}}+\frac{f_{k}}{f_{0}}\right)}{\left(1 \mp \frac{b_{k} f_{k}}{b_{0} f_{0}}\right)} \frac{a_{k}}{a_{0}}\right)\left(\frac{\frac{b_{j}}{b_{0}}+\frac{f_{j}}{f_{0}}}{\left(1 \mp \frac{b_{j} f_{j}}{b_{0} f_{0}}\right)}+\frac{a_{j}}{a_{0}}\right) \mathbf{e}_{j} .
\end{aligned}
$$

The product $(\stackrel{o}{\alpha} \stackrel{o}{\beta}) \stackrel{o}{\varphi}$ given by (3.4) and the product $\stackrel{o}{\alpha}(\stackrel{o}{\beta} \stackrel{o}{\varphi})$ given by (3.5) are clearly not equal for arbitrary scator coefficients. In appendix A.1, particular scator coefficients when associativity holds are evaluated in this and subsequent cases. If, in addition to $\frac{a_{l} b_{l}}{a_{0} b_{0}}= \pm 1$, another factor $\frac{a_{m} b_{m}}{a_{0} b_{0}}= \pm 1, m \neq l$, then $\stackrel{o}{\alpha} \stackrel{o}{\beta}=\stackrel{o}{0}$ and $(\stackrel{o}{\alpha} \stackrel{o}{\beta}) \stackrel{o}{\varphi}$ is also zero. However, this condition does not make the product $\stackrel{o}{\alpha}(\stackrel{o}{\beta} \stackrel{o}{\varphi})$ given by $(3.5)$ equal to zero. Non associativity due to zero products is a particular case of non associativity due to products with zero scalar component. Since $\stackrel{o}{0} \in \mathbb{S}_{0}^{1+n}$, they need not be treated separately.

Subcase 1.2 Two product pairs have null scalar component, $\stackrel{o}{\alpha} \stackrel{o}{\beta}, \stackrel{o}{\beta} \stackrel{o}{\varphi} \in \mathbb{S}_{0}^{1+n}, \stackrel{o o}{\alpha} \stackrel{\rho}{\varphi} \in \mathbb{S}_{\neq 0}^{1+n}$.

Product $\stackrel{o}{\alpha}(\stackrel{o}{\beta} \stackrel{o}{\varphi})$. Let the zero scalar in the $\stackrel{o}{\beta} \stackrel{o}{\varphi}$ product originate due to the $p^{t h}$ factor, $\left(1 \mp \frac{b_{p} f_{p}}{b_{0} f_{0}}\right)=0$. The subsequent product with an arbitrary scator $\stackrel{o}{\alpha}$ with non vanishing scalar is then given by (1.2b),

$$
\stackrel{o}{\alpha}(\stackrel{o}{\beta} \stackrel{o}{\varphi})=b_{0} f_{0} \prod_{k \neq p}^{n}\left(1 \mp \frac{b_{k} f_{k}}{b_{0} f_{0}}\right)\left(\frac{b_{p}}{b_{0}}+\frac{f_{p}}{f_{0}}\right)\left(\mp a_{p}+a_{0} \mathbf{e}_{p} \mp \sum_{j \neq p}^{n}\left(\frac{a_{p} a_{j}}{a_{0}}\right) \mathbf{e}_{j}\right) .
$$

Comparison of Eqs. (3.4) and (3.6) shows that these two expressions are different either for $l=p$ or $l \neq p$, for otherwise arbitrary scator coefficients.

Subcase 1.3 All three product pairs have null scalar component, $\stackrel{o}{\alpha} \stackrel{o}{\beta}, \stackrel{o}{\beta} \stackrel{o}{\varphi}, \stackrel{o}{\alpha} \varphi \in \mathbb{S}_{0}^{1+n}$. The derivation involves, in addition to the previous case, a zero scalar due to the $q^{t h}$ factor, $\left(1 \mp \frac{a_{q} f_{q}}{a_{0} f_{0}}\right)=0$ of the $\stackrel{o}{\alpha} \stackrel{o}{\varphi}$ product. The procedure is analogous to the previous subcase.

Case 2. Product of two scator factors with non zero additive scalar, $\stackrel{o}{\alpha}, \stackrel{o}{\beta} \in \mathbb{S}_{\neq 0}^{1+n}$, and one factor with zero scalar, $\stackrel{o}{\varphi} \in \mathbb{S}_{m}^{1+n}$.

Subcase 2.1 Only one product pair has a vanishing scalar $\stackrel{o}{\alpha} \stackrel{o}{\beta} \in \mathbb{S}_{l}^{1+n}, \stackrel{o}{\beta} \stackrel{o}{\varphi}, \stackrel{o}{\alpha} \stackrel{o}{\varphi} \in \mathbb{S}_{\neq 0}^{1+n}$.

$\operatorname{Product}(\stackrel{o}{\alpha} \stackrel{o}{\beta}) \stackrel{o}{\varphi}$. If $\stackrel{o}{\alpha} \stackrel{o}{\beta} \in \mathbb{S}_{\neq 0}^{1+n}$ and $\stackrel{o}{\alpha} \stackrel{o}{\beta} \in \mathbb{S}_{l}^{1+n}$, the product is given by (3.2). The product of this result times $\stackrel{o}{\varphi}=f_{m} \mathbf{e}_{m} \in \mathbb{S}_{m}^{1+n}$ is from $(1.2 \mathrm{c})$,

$$
(\stackrel{o}{\alpha} \stackrel{o}{\beta}) \stackrel{o}{\varphi}=\mp a_{0} b_{0} f_{m} \prod_{k \neq l}^{n}\left(1 \mp \frac{a_{k} b_{k}}{a_{0} b_{0}}\right)\left(\frac{a_{l}}{a_{0}} \pm \frac{a_{0}}{a_{l}}\right) \delta_{l m} .
$$

Product $\stackrel{o}{\alpha}(\stackrel{o}{\beta} \stackrel{o}{\varphi})$. The product $\stackrel{o}{\beta} \stackrel{o}{\varphi}$, since $b_{m} \neq 0$ is obtained from $(1.2 \mathrm{~b})$. The product with $\stackrel{o}{\alpha}$, if $l \neq m$, is

$$
\stackrel{o}{\alpha}(\stackrel{o}{\beta} \stackrel{o}{\varphi})=\mp a_{0} b_{m} f_{m} \prod_{k \neq l, m}^{n}\left(1 \mp \frac{a_{k} b_{k}}{a_{0} b_{0}}\right)\left(1+\frac{a_{m} b_{0}}{a_{0} b_{m}}\right)\left(\frac{a_{l}}{a_{0}} \pm \frac{a_{0}}{a_{l}}\right) \mathbf{e}_{l} .
$$

This single director component scator differs from (3.7), since the latter has no director component. If $l=m$,

$$
\stackrel{o}{\alpha}(\stackrel{o}{\beta} \stackrel{o}{\varphi})=\mp a_{0} b_{l} f_{l}\left(1 \pm \frac{a_{l}^{2}}{a_{0}^{2}}\right) \prod_{k \neq l}^{n}\left(1 \mp \frac{a_{k} b_{k}}{a_{0} b_{0}}\right) \mp a_{0} b_{l} f_{l}\left(1 \pm \frac{a_{l}^{2}}{a_{0}^{2}}\right) \sum_{j \neq l}^{n}\left[\prod_{k \neq j, l}^{n}\left(1 \mp \frac{a_{k} b_{k}}{a_{0} b_{0}}\right)\left(\frac{a_{j}}{a_{0}}+\frac{b_{j}}{b_{0}}\right)\right] \mathbf{e}_{j} .
$$

This expression also differs from (3.7). Subcases 2.2 where two product pairs have a vanishing scalar $\stackrel{o}{\alpha} \stackrel{o}{\beta}, \stackrel{o}{\beta} \stackrel{o}{\varphi} \in \mathbb{S}_{0}^{1+n}, \stackrel{o o}{\alpha} \stackrel{\varphi}{\varphi} \in \mathbb{S}_{\neq 0}^{1+n}$ and 2.3 where all three product pairs have null scalar component, $\stackrel{o}{\alpha} \stackrel{o}{\beta}, \stackrel{o}{\beta} \stackrel{o}{\varphi}, \stackrel{o}{\alpha} \stackrel{o}{\varphi} \in \mathbb{S}_{0}^{1+n}$ are similarly tackled. 
Case 3. Product of one scator factor with non zero scalar, $\stackrel{o}{\varphi} \in \mathbb{S}_{\neq 0}^{1+n}$, and two factors with zero additive scalar, $\stackrel{o}{\alpha}, \stackrel{o}{\beta} \in \mathbb{S}_{0}^{1+n}$. Subcase $3.1 \mathrm{l} \neq \mathrm{m}$

Product $(\stackrel{o}{\alpha} \stackrel{o}{\beta}) \stackrel{o}{\varphi}$. The product of two scators $\stackrel{o}{\alpha}, \stackrel{o}{\beta} \in \mathbb{S}_{0}^{1+n}$ with different non zero director component, say $\stackrel{o}{\alpha}=a_{m} \mathbf{e}_{m}, \stackrel{o}{\beta}=b_{l} \mathbf{e}_{l}$, from $(1.2 \mathrm{c})$, is zero. Their product with an arbitrary scator $\stackrel{o}{\varphi}$ is zero, $\left(a_{m} \mathbf{e}_{m} b_{l} \mathbf{e}_{l}\right) \stackrel{o}{\varphi}=0$.

Product $\stackrel{o}{\alpha}(\stackrel{o}{\beta} \stackrel{o}{\varphi})$. The product of $\stackrel{o}{\varphi} \in \mathbb{S}_{\neq 0}^{1+n}$ times a single director component scator $\stackrel{o}{\beta}=b_{l} \mathbf{e}_{l}$, obtained from $(1.2 \mathrm{~b})$, is

$$
b_{l} \mathbf{e}_{l} \stackrel{o}{\varphi}=b_{l}\left(\mp f_{l}+f_{0} \mathbf{e}_{l} \mp\left(\frac{f_{l} f_{m}}{f_{0}}\right) \mathbf{e}_{m} \mp \sum_{j \neq l, m}^{n}\left(\frac{f_{l} f_{j}}{f_{0}}\right) \mathbf{e}_{j}\right),
$$

where the $l^{t h}$ and $m^{t h}$ terms are shown explicitly. Multiplication by $\stackrel{o}{\alpha}=a_{m} \mathbf{e}_{m}$, recalling that $m \neq l$, again from (1.2b) is,

$$
\stackrel{o}{\alpha}(\stackrel{o}{\beta} \stackrel{o}{\varphi})=a_{m} \mathbf{e}_{m}\left(b_{l} \mathbf{e}_{l}^{o} \stackrel{\varphi}{\varphi}\right)=a_{m} b_{l}\left(\left(\frac{f_{l} f_{m}}{f_{0}}\right) \mp f_{l} \mathbf{e}_{m} \mp f_{m} \mathbf{e}_{l}+\sum_{j \neq l, m}^{n}\left(\frac{f_{l} f_{m} f_{j}}{f_{0}^{2}}\right) \mathbf{e}_{j}\right) .
$$

This expression is not zero and thus differs from the product $(\stackrel{o}{\alpha} \stackrel{o}{\beta}) \stackrel{o}{\varphi}$.

Subcase 3.2 If $l=m$, the product $(\stackrel{o}{\alpha} \stackrel{o}{\beta})=\left(a_{m} \mathbf{e}_{m} b_{l} \mathbf{e}_{l}\right)=a_{l} b_{l}$ has non zero scalar component. The product of $\stackrel{o}{\alpha} \stackrel{o}{\beta}$ with $\stackrel{o}{\varphi} \in \mathbb{S}_{\neq 0}^{1+n}$ gives a scator with non zero scalar component. Thus, a product pair with zero scalar component cannot be achieved in this subcase.

Case 4. Product of three scator factors with zero additive scalar $\stackrel{o}{\alpha}, \stackrel{o}{\beta}, \stackrel{o}{\varphi} \in \mathbb{S}_{0}^{1+n}$.

If the scator $\stackrel{o}{\varphi}$ also has vanishing scalar component, the product definition (1.2c) has to be used throughout. For $l \neq m$, $(\stackrel{o}{\alpha} \stackrel{o}{\beta}) \stackrel{o}{\varphi}=$ $\left(a_{l} \mathbf{e}_{l} b_{m} \mathbf{e}_{m}\right)\left(f_{m} \mathbf{e}_{m}\right)=0$ and $\stackrel{o}{\alpha}(\stackrel{o}{\beta} \stackrel{o}{\varphi})=\left(a_{l} \mathbf{e}_{l}\right)\left(b_{m} \mathbf{e}_{m} f_{m} \mathbf{e}_{m}\right)=a_{l} b_{m} f_{m} \mathbf{e}_{m}$. Therefore, this product is not associative if two director components have different subindices.

In accordance with Theorems 2.1 and 3.1, non vanishing additive scalar components in all possible product pairs is a sufficient condition for scator associativity while at least one product pair with vanishing scalar component is a necessary condition for non associativity.

\section{Wavefunction collapse}

The quantum mechanical wave function, according with the prevailing Copenhagen view, contains probabilistic information regarding the state of a system. In a simplified scheme, when an observation is made, only one state is detected from the superposition of all possible states. This process, whereby the probability of all but one state become zero and the remaining state becomes a certainty, is the collapse or reduction of the wave function. Continuous spectrum operators such as position or momentum do not collapse to a single state, but to a combination of eigenstates within a spread of eigenvalues given by the nature of the measurement. The wave function reduction, whether to a single state or a range of closely packed states determines the state of the system up to the precision imposed by the uncertainty principle. Let us recall the formal description of the two processes involved in the evolution of a quantum system as a preliminary to the scator description. In the Schrödinger representation, for a time independent Hamiltonian $\mathscr{H}$, the time evolution of the wave function $|\psi\rangle$ is given by a unitary time evolution propagator $\mathscr{U}$, so that $|\psi(t)\rangle=\mathscr{U}|\psi(0)\rangle$. The wave function is written as a superposition of energy eigenfunctions $|\psi\rangle=\sum_{j=1}^{n} c_{j}\left|\psi_{j}\right\rangle$, such that $\mathscr{H}\left|\psi_{j}\right\rangle=\mathscr{E}_{j}\left|\psi_{j}\right\rangle$. The set of eigenfunctions and their corresponding propagators in matrix form are $\psi(t)=\mathbf{U} \psi(0)$. If the systems evolves from 0 to $t_{1}$ and then from $t_{1}$ to $t_{2}$ and so on, the evolution may be written as a product of matrices $\psi(t)=\mathbf{U}_{f} \cdots \mathbf{U}_{2} \mathbf{U}_{1} \psi(0)$, provided that there are no state reductions in the process. This picture is broken down when the operator $\mathbf{R}$, reduces the wavefunction to a single $j$ state, $\mathbf{R} \psi(t)=\left|\psi_{j}\right\rangle$. These two distinct procedures $\mathbf{U}$ and $\mathbf{R}$, required in order to describe a quantum system, are referred to as the quantum measurement problem [1]. Different approaches have been proposed to unify these two mechanisms under the generic name of dynamical reduction models [2]. The aim of the following scator description is to contribute towards the formalization of a reduction model.

Allow for the quantum wave function to be described by a scator function $\stackrel{o}{\psi}(t)$ instead of a column vector. Each eigenfunction $\left|\psi_{j}\right\rangle$ is associated with a scator's director component $\left|\psi_{j}\right\rangle \rightarrow \mathbf{e}_{j}$. The wavefunction eigenstates are described by the director components of a scator function $|\psi\rangle+c_{0}=\stackrel{o}{\psi}=c_{0}+\sum_{j=1}^{n} c_{j} \mathbf{e}_{j}$. This is certainly a huge formal leap, and it is not certain that it is possible, but let us continue with the sketch of the procedure in the scator formalism. Notice, that we must add a scalar function $c_{0}$ for $n \geq 2$, otherwise, the scator is not in $\mathbb{S}^{1+n}$. Since all the possible wave eigenfunctions have been ascribed to the director components of the scator, we have freedom to establish the value of the scalar function. This function can be associated with the time variable. The time evolution of the system is modeled by the product of unitary scators $\stackrel{o}{\Upsilon}_{k}$ times the system's wavefunction

$$
\stackrel{o}{\psi}(t)=\stackrel{o}{\Upsilon}_{f} \cdots \stackrel{o}{\Upsilon} \stackrel{o}{\Upsilon}_{1} \stackrel{o}{\psi}(0),
$$

where $\stackrel{o}{\Upsilon}_{k} \in \mathbb{S}^{1+n}$ for all $k$ from the initial to the final time interval $f$ and $\stackrel{o}{\psi}(0) \in \mathbb{S}^{1+n}$. Unitary scators are obtained from the main involution of scator algebra; The conjugate of the scator $\stackrel{o}{\varphi}=f_{0}+\sum_{j=1}^{n} f_{j} \mathbf{e}_{j}$, is given by $\stackrel{o *}{\varphi}=f_{0}-\sum_{j=1}^{n} f_{j} \mathbf{e}_{j}$, that is, all its director components reverse sign while the scalar component remains unaltered. The product $\stackrel{o}{\varphi} \varphi^{*}=\|\stackrel{o}{\varphi}\|^{2}$ is a real number that establishes a magnitude or order parameter. Scators of the form $\stackrel{o}{\varphi} /\|\stackrel{o}{\varphi}\|$ constitute the set of unit magnitude scators [6]. Let the scalar component represent time evolution and wavefunction reduction, the latter taking place when the scalar component becomes zero, as the reader may have already guessed. When the scator product yields a zero scalar additive component, all director components must be zero with one possible exception as was shown in subsection 3.1. Since each director component has been chosen to represent an eigenstate, the product then models a wave function 
collapse, for in this circumstance only one eigenfunction subsists. Depending on which is the $l^{\text {th }}$ factor that fulfills condition (3.1), it is the $l^{\text {th }}$ eigenfunction from the 1 to $n$ eigenfunctions that survives. Therefore, an $\mathbf{R}$ measurement process is attained.

If the products have a non vanishing additive scalar, all director components prevail and since each represents a wave eigenfunction, a superposition of all of them is obtained. The products are then associative (Theorem 2.1) and can be performed with any precedence or grouped together, i.e. $\stackrel{o}{\Upsilon}_{3}\left(\stackrel{o}{\Upsilon}_{2}\left(\Upsilon_{1}^{o} \stackrel{o}{\psi}(0)\right)\right)=\left(\stackrel{o}{\Upsilon}_{3} \Upsilon_{2}^{o} \Upsilon_{1}^{o}\right) \stackrel{o}{\psi}(0)=\stackrel{o}{\Upsilon}_{i} \stackrel{o}{\psi}(0)$. The way these products are associated, represent an evolution that can be traced in several steps, grouped with any precedence or the evolution performed in one single step. Whether these products commute, depend on the operators involved in the scator coefficients. This is analogous to the usual quantum description with complex algebra. Complex algebra is commutative, but the complex numbers involve operators in quantum mechanics that may or may not commute. Physically, time evolution $\mathbf{U}$ processes are then described when the scator products have non vanishing additive scalar.

The scator product provides a unified description of the $\mathbf{U}$ and $\mathbf{R}$ processes. For example, $\left(\Upsilon_{4}^{o} \Upsilon_{i}^{o}\right) \in \mathbb{S}_{0}^{1+n}$ represents a collapse and the product $\stackrel{o}{\Upsilon}_{5}\left(\Upsilon_{\Upsilon}^{o} \stackrel{o}{\Upsilon}_{i}\right) \in \mathbb{S}_{\neq 0}^{1+n}$ the subsequent $\mathbf{U}$ evolution. When the product is not associative, it reflects the fact that the subsequent evolution of the system is altered by the measurement or collapse of the wave function. After a collapse, the time evolution of the system is again described by another wave function, where the initial conditions are given by the state of the collapsed system. Notice that the product of the collapsed system, say in the $\left(\stackrel{o}{\Upsilon}_{4} \stackrel{o}{\Upsilon}_{i}\right)=a_{l} \mathbf{e}_{l}$ state, regains a finite probability for all possible states when its product is taken with a unitary evolution scator $\stackrel{o}{\Upsilon}_{5}=\stackrel{o}{\beta}$, with arbitrary non vanishing director components (equal to the number of possible states) given by the product definition (1.2b). Therefore, one and the same mathematical procedure, the scator product, is used to describe the complete time evolution including the possible wave function reductions of the physical system. This scheme is in the vein of the Penrose proposal [15, Sec.22.1, p.529] whereby the same mathematical object, the scator product in this approach, describes the $\mathbf{U}$ and $\mathbf{R}$ processes. It could be argued that the two distinct procedures have only been deferred to the two definitions of the scator product (1.2a) and (1.2b), depending on whether the scalar component of $\stackrel{o}{\alpha}$ vanishes or not. However, it is possible to obtain the product (1.2b) from (1.2a) through a limit. In order to remain within the $\mathbb{S}^{1+n}$ set, the limit of all but one of the director components should be taken first and thereafter the scalar component limit should be taken [7]. The $\stackrel{o}{\alpha}$ director component limits from (1.2a) is

$$
\lim _{a_{k \neq l} \rightarrow 0}(\stackrel{o o}{\alpha} \stackrel{o}{\alpha})=\left(a_{0} b_{0} \mp a_{l} b_{l}\right)+\left(a_{l} b_{0}+a_{0} b_{l}\right) \mathbf{e}_{l}+\sum_{j \neq l}^{n}\left(a_{0} b_{0} \mp a_{l} b_{l}\right)\left(\frac{b_{j}}{b_{0}}\right) \mathbf{e}_{j}
$$

Thereafter, the scalar component limit is

$$
\lim _{a_{0} \rightarrow 0}\left(\lim _{a_{k \neq l} \rightarrow 0}(\stackrel{o o}{\alpha \beta})\right)=\mp a_{l} b_{l}+a_{l} b_{0} \mathbf{e}_{l} \mp \sum_{j \neq l}^{n} a_{l} b_{l}\left(\frac{b_{j}}{b_{0}}\right) \mathbf{e}_{j} .
$$

But this result is identical to (1.2b), thus this latter definition is a removable singularity of the product function (1.2a) with arbitrary but non vanishing scalar components. Another asset of this formalism is that no 'tails' are present when the collapse takes place. The tail problem arises because finite, albeit small, amplitude probabilities for states other than the reduced state are present in some proposals, such as the GRW scheme [2]. In the present scheme, all states different from the collapsed state are strictly zero. There are other issues that require careful assessment to confirm whether this proposal is plausible. Two ingredients are essential to achieve a unified dynamical scheme, nonlinearity and an stochastic process. Here, the nonlinearity is ultimately due to the scator product definition. However, an stochastic process still needs to be incorporated. Nonetheless, according to this initial assessment, scator algebra seems to be a promising route for a unified description of quantum dynamics.

\section{Conclusions}

Product associativity in either real or imaginary scator algebras depends on whether the additive scalar component of any product pair is zero. The scator product is associative in $\mathbb{S}^{1+n}$ if all possible product pairs have a non vanishing additive scalar component. Recall that the scator set $\mathbb{S}^{1+n}$ where the product is defined in the additive representation, is the subset of $\mathbb{R}^{1+n}$ where the additive scalar is different from zero if two or more director components are not zero. The scator product is in general not associative in the additive representation in $\mathbb{S}^{1+n}$ for dimensions with $n>1$, if one or more of the possible product pairs has a vanishing additive scalar component. At least one product pair with zero scalar component is a necessary condition for non associativity. These assertions are embodied in theorems 2.1 and 3.1.

The additive scalar component of any product pair is the decisive parameter in order to establish whether the scator product is associative or not. The stronger condition stated in previous communications for lack of associativity, if all components of any product pair are zero (i.e. a zero scator), has been included in the zero additive scalar component necessary condition (i.e. a scator with possibly one non vanishing director component).

The main involution is scator algebras is conjugation [6]. The conjugate of a scator $\stackrel{o}{\varphi}=f_{0}+\sum_{j=1}^{n} f_{j} \mathbf{e}_{j}$, is defined by the negative of its director components while the scalar component remains unchanged, $\stackrel{o^{*}}{\varphi} \equiv f_{0}-\sum_{j=1}^{n} f_{j} \mathbf{e}_{j}$. The magnitude of a scator is defined by the positive square root of the scator times its conjugate $\|\stackrel{o}{\varphi}\|=\sqrt{{\stackrel{o}{\varphi o^{*}}}^{\varphi}}$. From the product definition, clearly \|\|$^{o} \|^{2} \in \mathbb{R}$.

Corollary 5.1. In $\mathbb{S}^{1+n}$, the magnitude of two or more scator products is equal to the product of their magnitudes if the products are associative.

Proof. The square magnitude of the product of two scators $\stackrel{o}{\alpha}, \stackrel{o}{\beta}$, according to the magnitude definition is $\|\stackrel{o o}{\alpha \beta}\|^{2}=(\stackrel{o}{\alpha \beta})(\stackrel{o * * *}{\alpha} \stackrel{o *}{\beta})$. Since

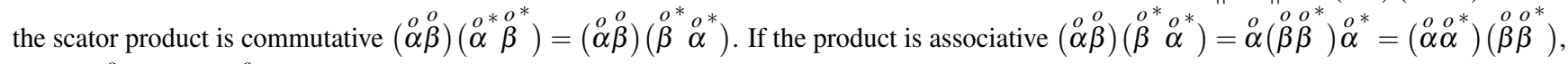
thus $\|\stackrel{o}{\alpha} \beta\|=\|\stackrel{o}{\alpha}\|\|\stackrel{o}{\beta}\|$. 
The magnitude of the scator products is equal to the product of the scator magnitudes if all possible product pairs have a non vanishing additive scalar component. Therefore, the $\stackrel{o}{\alpha} \beta \neq 0$ condition stated in Lemma 4.1 [12], is now corrected and embraced in the condition stated in this corollary. Notice that $\stackrel{o}{\varphi}+\stackrel{o *}{\varphi}=2 f_{0}$, thus a scator product with non zero scalar component can be stated in terms of the involution as $\stackrel{o}{\alpha} \beta+\stackrel{o *}{\alpha} \stackrel{o *}{\beta} \neq 0$.

\section{A. Appendix: Associativity exceptions}

There are three generic conditions where the scalar component of a product pair is zero but associativity holds: i) The $\mathbb{S}^{1+1_{j}}$ subsets; ii) Two scator factors are linearly dependent on all director coefficients except for the two that produce a zero scalar ; iii) All product pairs give zero products.

The foremost exception are scator sets with only one director component

$$
\mathbb{S}^{1+1_{j}}=\left\{\stackrel{o}{\varphi}=f_{0}+\sum_{k=1}^{n} f_{k} \mathbf{e}_{k}, \in \mathbb{S}^{1+n}: f_{k}=0, \text { for any } k \neq j\right\}
$$

Numbers in the $\mathbb{S}^{1+1_{j}}$ subset are of the form $\stackrel{o}{\varphi}=f_{0}+f_{j} \mathbf{e}_{j}$, these subsets are isomorphic to the complex field for each $j$, [10, Proposition 4.12]. The scator product definition (1.2a)-(1.2c) can then be grouped in the usual single product definition for complex numbers (or hyperbolic numbers). The scator product is thus associative in $\mathbb{S}^{1+1_{j}}$ even if a product pair has zero scalar component.

In the proof of Theorem 3.1, non associativity was analyzed in various cases where different expressions were obtained depending on the precedence of evaluation. These expressions are forced here to be equal by imposing the necessary conditions on the scator coefficients.

\section{A.1. Linear dependence except for $l^{\text {th }}$ component (Case 1)}

The product in subcase 1.1 of Theorem 3.1 is not associative unless $(\stackrel{o}{\alpha} \beta) \stackrel{o}{\varphi}$ given by (3.4) and $\stackrel{o}{\alpha}(\stackrel{o}{\beta} \stackrel{o}{\varphi})$ given by (3.5) are equal. Equating the scalar terms imposes

$$
\prod_{k \neq l}^{n}\left(1 \mp \frac{a_{k} b_{k}}{a_{0} b_{0}}\right)=\prod_{k \neq l}^{n}\left(1 \mp \frac{a_{k} b_{k}}{a_{0} b_{0}} \mp \frac{b_{k} f_{k}}{b_{0} f_{0}} \mp \frac{a_{k} f_{k}}{a_{0} f_{0}}\right) .
$$

The $\mathbf{e}_{j}$ components in (3.4) and (3.5) are equal only if, in addition to the above condition,

$$
\frac{f_{j}}{f_{0}}=\frac{\left(\frac{a_{j}}{a_{0}}+\frac{b_{j}}{b_{0}}+\frac{f_{j}}{f_{0}} \mp \frac{a_{j}}{a_{0}} \frac{b_{j} f_{j}}{b_{0} f_{0}}\right)}{\left(1 \mp \frac{b_{j} f_{j}}{b_{0} f_{0}} \mp \frac{a_{j} b_{j}}{a_{0} b_{0}} \mp \frac{a_{j} f_{j}}{a_{0} f_{0}}\right)}
$$

for all $j \neq l$ from 1 to $n$. This equality holds provided that i) $\frac{a_{j}}{a_{0}}+\frac{b_{j}}{b_{0}} \neq 0$ and $f_{j}^{2}=\mp f_{0}^{2}$, but this condition cannot be fulfilled for imaginary scators except if $f_{j}=f_{0}=0$, but then $\stackrel{o}{\varphi} \in \mathbb{S}_{0}^{1+n}$, contrary to the initial premise. For real scators $f_{j}^{2}=f_{0}^{2}$, implies that the scator lies in the null geodesic surface [12]. ii) If $\frac{a_{j}}{a_{0}}+\frac{b_{j}}{b_{0}}=0$, then $b_{j}=-b_{0} \frac{a_{j}}{a_{0}}$. The product of scators

$$
\stackrel{o}{\alpha}=a_{0}\left(1+\sum_{j=1}^{n} \frac{a_{j}}{a_{0}} \mathbf{e}_{j}\right), \quad \stackrel{o}{\beta}=b_{0}\left(1-\sum_{j \neq l}^{n} \frac{a_{j}}{a_{0}} \mathbf{e}_{j} \pm \frac{a_{0}}{a_{l}} \mathbf{e}_{l}\right), \quad \stackrel{o}{\varphi}=f_{0}+\sum_{j=1}^{n} f_{j} \mathbf{e}_{j},
$$

is then associative although $\stackrel{o}{\alpha} \beta \in \mathbb{S}_{0}^{1+n}$. The scators $\stackrel{o}{\alpha}$ and $\stackrel{o}{\beta}$ are linearly dependent on all $\mathbf{e}_{j}$ coefficients except for the $\mathbf{e}_{l}$ coefficient, where $a_{l} b_{l}= \pm a_{0} b_{0}$ produces a product $\stackrel{o}{\alpha} \beta$ with zero scalar component. Associativity in subcase 1.2 of Theorem 3.1 is obtained by imposing the equality of equations (3.4) and (3.6). This equality again requires linear dependence on all but one of the director components.

\section{A.2. Zero product pairs (Cases 2, 3 and 4)}

Subcase 2.1 If $l \neq m$, equations (3.7) and (3.8), require the scalar component to be equal to the director component $\mathbf{e}_{l}$, a condition that can only be satisfied if both coefficients are zero. A factor in the product $\prod_{k \neq l, m}^{n}\left(1 \mp \frac{a_{k} b_{k}}{a_{0} b_{0}}\right)$ of equation (3.8) then has to be zero. Hence $(\stackrel{o}{\alpha} \stackrel{o}{\beta}) \stackrel{o}{\varphi}=\stackrel{o}{\alpha}(\stackrel{o}{\beta} \stackrel{o}{\varphi})=\stackrel{o}{\beta}(\stackrel{o}{\alpha} \stackrel{o}{\varphi})=0$. Subcase 2.1 with $l=m$, as well as subcase 2.2 and cases 3 and 4 are similarly obtained and again associativity holds when all product pairs are zero.

\section{Acknowledgement}

This work was partially developed while on sabbatical leave at the Instituto de Investigaciones en Matemáticas Aplicadas y en Sistemas, Universidad Nacional Autónoma de México, Ciudad Universitaria, CDMX, México.

\section{References}

[1] V. Allori, S. Goldstein, R. Tumulka, and N. Zanghì, On the Common Structure of Bohmian Mechanics and the Ghirardi-Rimini-Weber Theory, British Journal for the Philosophy of Science 59 (2008), no. 3, 353-389.

[2] A. Bassi and G. C. Ghirardi, Dynamical reduction models, Physics Reports 379 (2003), no. 5-6, $257-426$. 
[3] W. E. Baylis and J. D. Keselica, A classical spinor approach to the quantum/classical interface, Canadian Journal of Physics 86 (2008), no. 4, 629-634

[4] F. Catoni, D. Boccaletti, R. Cannata, V. Catoni, E. Nichelatti, and P. Zampetti, The Mathematics of Minkowski Space-Time, Frontiers in Mathematics, no. 2, Birkhauser Verlag, 2008.

[5] M. Fernández-Guasti, Time and space transformations in a scator deformed Lorentz metric, European Physical Journal - Plus 129 (2014), no. 195, 1-10.

[6] A non-distributive extension of complex numbers to higher dimensions, Adv. Appl. Clifford Algebras 25 (2015), 829-849.

[7] Imaginary Scators Bound Set Under The Iterated Quadratic Mapping In 1+2 Dimensional Parameter Space, Int. J. of Bifurcation and Chaos 26 (2016), no. 1, 1630002.

[8] Differential quotients in elliptic scator algebra, Math. Meth. App. Sci. (2018), 1-14, DOI:10.1002/mma.4933.

[9] M. Fernández-Guasti and F. Zaldívar, A hyperbolic non distributive algebra in 1+2 dimensions, Adv. Appl. Clifford Algebras 23 (2013), no. 3, 639-653.

[10] - An elliptic non distributive algebra, Adv. Appl. Clifford Algebras 23 (2013), no. 4, 825-835.

[11] Multiplicative representation of a hyperbolic non distributive algebra, Adv. Appl. Clifford Algebras 24 (2014), no. 3, 661-674.

[12] _ Hyperbolic superluminal scator algebra, Adv. Appl. Clifford Algebras 25 (2015), no. 2, 321-335.

[13] D. Hestenes, New foundations for classical mechanics, Kluwer, 1990.

[14] A. Kobus and J. L. Cieśliński, On the Geometry of the Hyperbolic Scator Space in 1+2 Dimensions, Advances in Applied Clifford Algebras 27 (2017), no. 2, 1369-1386.

[15] R. Penrose, The Road to reality, Knopf, 2005. 